\title{
Determinants of hypofibrinolysis in patients with digestive tract cancer
}

\author{
Katarzyna Gronostaj, Piotr Richter, Wojciech Nowak, Anetta Undas \\ Institute of Cardiology, Jagiellonian University Medical College, Krakow, Poland
}

Gastroenterology Rev 2016; 11 (2): 104-110

DOI: $10.5114 / p g .2016 .57619$

Key words: digestive tract cancer, fibrinolysis, platelet activation, thrombin generation, thrombin activatable fibrinolysis inhibitor.

Address for correspondence: Anetta Undas MD, PhD, Institute of Cardiology, Jagiellonian University Medical College, 80 Pradnicka St, 31-202 Krakow, Poland, phone: +48 1261430 04, fax: +48 1242339 00, e-mail: mmundas@cyf-kr.edu.pl

\begin{abstract}
Introduction: Recently, we demonstrated that digestive tract cancer (DTC) is associated with reduced fibrin clot permeability and impaired fibrinolysis.

Aim: We investigated determinants of fibrinolysis in DTC patients.

Material and methods: In 44 consecutive patients with DTC and 47 controls matched for age, sex, and cardiovascular risk, we evaluated fibrinolysis proteins, platelet activation markers, thrombin formation, together with plasma clot lysis time assays in the absence (CLT) and presence of carboxypeptidase potato inhibitor (CLT CPI) that blocks thrombin activatable fibrinolysis inhibitor (TAFI).

Results: In the DTC group CLT (by 22.3\%) and CLT CPI (by 27.4\%) were longer compared with controls. The DTC patients had higher plasma fibrinolysis inhibitors, plasminogen activator inhibitor 1 (PAI-1) (by 18.2\%), TAFI activity (by 17.3\%), and antigen (by 11.2\%). The patients had markedly increased platelet markers - soluble CD40 ligand (by 338\%) and P-selectin (by 97\%), together with von Willebrand factor (VWF) antigen (by 61\%). Thrombin-antithrombin complexes (TAT) (by 48.7\%) and soluble thrombomodulin (STM) (by 17.2\%) were also increased in the DTC group (all $p<0.05$ ). Patients with high-grade tumours $(n=26)$ compared with remainders $(n=18)$ had longer CLT, higher tissue-type plasminogen activator antigen, both TAFI antigen and activity levels, VWF, and STM. Multiple regression analysis after adjustment for potential confounders showed that independent predictors of CLT in DTC patients were TAT, TAFI activity, and VWF. The only independent predictor of CLT CPI was TAT.

Conclusions: Hypofibrinolysis in DTC patients is largely driven by enhanced thrombin generation, TAFI, and endothelial injury.
\end{abstract}

\section{Introduction}

Thrombosis has a significant impact on the morbidity and mortality in cancer patients [1]. The risk of a thrombotic event in patients with malignancy depends on several factors, including the type of cancer, its clinical stage and grade, concomitant disease, and treatment. Elevated plasma D-dimer levels, which reflect a global activation of blood coagulation and fibrinolysis, are commonly observed in cancer patients and may predict poor prognosis [2, 3]. Global fibrinolytic capacity, detected with the amount of D-dimer produced by tissue-type plasminogen activator (tPA) from standard fibrin pellets, has also been shown to be increased in both metastatic and non-metastatic disease of patients with colorectal cancer, reflecting a progression to overt disseminated intravascular coagulation [4]. On the other hand, several reports have demonstrated increased circulating levels of fibrinolysis inhibitors, including plasminogen activator inhibitor (PAI-1) [5] and thrombin activatable fibrinolysis inhibitor (TAFI), in cancer patients, including those with lung and gastric cancer [6-8], which suggests impaired fibrinolysis in this disease. The PAI-1 is a major physiological inhibitor of TPA, while TAFI is a proenzyme, which is converted to its active form by thrombin in complex with thrombomodulin, and activated TAFI cleaves C-terminal lysine and arginine residues from partially degraded fibrin, thereby preventing tPA and plasminogen binding, which is necessary for efficient plasminogen activation [9]. $\alpha_{2}$-Antiplasmin $\left(\alpha_{2} A P\right)$ rapidly inhibits plasmin in solution, but not when bound to fibrin. Both TAFI and $\alpha_{2} A P$ are important in the inhibition and downregulation of plasmin [9].

Higher antigen levels of urokinase-type plasminogen activator (U-PA), UPA receptor (UPAR), PAI-1, and 
plasminogen activator inhibitor-2 (PAl-2) were found in colorectal cancer (CRC) tissue compared with normal tissue [10]. However, this mechanism appears to be of negligible importance for systemic fibrinolysis measured in plasma of venous blood.

Little is known about the links between circulating concentrations of fibrinolysis inhibitors and the stage of cancer. Langenskiold et al. reported a correlation between plasma PAI-1 and metastatic disease in CRC patients [11]. Fidan et al. found a positive association between plasma TAFI levels and lymph node invasion in gastric cancer patients [8]. It might be speculated that impaired fibrinolysis is more common among patients with advanced cancer.

Recently we have demonstrated that digestive tract cancer (DTC) patients present unfavourably altered clot properties, including faster clot formation, reduced clot permeability, and impaired susceptibility to lysis, which were evaluated in plasma of venous blood [12]. Patients with DTC had, among others, longer clot lysis time $(+17 \%)$ compared with controls, which were in part related to increased thrombin-antithrombin (TAT) complexes, a measure of thrombin generation, and plasma PAI-1 levels [12]. However, it is unclear whether other fibrinolysis inhibitors could contribute to hypofibrinolysis observed in cancer patients. The role of several proteins released from activated platelets and/or endothelial cells in impaired fibrinolysis measured in plasma of venous blood also remains to be elucidated.

\section{Aim}

Therefore, we decided to investigate which factors determine reduced plasma fibrin clot lysis in DTC patients.

\section{Material and methods}

\section{Subjects}

The DTC patients $(n=44)$ we investigated were previously described in detail [12]. In short, we enrolled consecutive patients with histologically confirmed DTC and compared them with 47 controls matched for age, sex, and cardiovascular risk in the case-control study. Sixteen patients were diagnosed with rectal cancer, 10 with colon cancer, 8 with gastric cancer, 8 with pancreatic cancer, and 2 with small bowel cancer. Only 3 subjects had stage I (based on the American Joint Committee on Cancer staging scheme [13]) disease, 4 had stage II, 19 had stage III, and 18 had stage IV disease. There were 3 patients with grade 1 tumours (G1, low grade), 15 patients with grade 2 (G2, intermediate grade), and 26 patients with grade 3 (G3, high grade) according to the World Health Organisation grading scheme. The exclusion criteria were: any active infection, renal dysfunc- tion (creatinine $>2 \mathrm{mg} / \mathrm{dl}$ ), hypo- and hyperthyroidism, previous myocardial infarction, stroke or another acute vascular event, venous thromboembolism (VTE), known bleeding diathesis, and current anticoagulant therapy except for low molecular weight heparin administered for the last time $12 \mathrm{~h}$ or more prior to sample collection.

The Jagiellonian University Bioethics Committee approved the study protocol. Written consent was obtained from each participant.

\section{Laboratory investigations}

Fasting blood was taken from the antecubital vein between 7 and 9 a.m. Routine laboratory tests were used to determine blood cell count, glucose, creatinine, protein, albumin, international normalised ratio (INR), activated partial thromboplastin time (APTT), C-reactive protein (CRP), and fibrinogen.

Plasma $\alpha 2$-antiplasmin $\left(\alpha_{2} A P\right)$ and plasminogen were measured by chromogenic assays (STA Stachrom $\alpha 2$-antiplasmin and STA Stachrom plasminogen, Diagnostica Stago). Plasma PAI-1 antigen and tPA antigen were measured by enzyme-linked immunosorbent assays (ELISAs, Hyphen BioMed, Neuville Sur-Oise, France). Measurement of TAFI antigen was performed with an ELISA (Chromogenix, Lexington, MA, USA). Plasma TAFI activity was measured by a chromogenic assay using the ACTICHROME ${ }^{\circledR}$ Plasma TAFI Activity Kit (American Diagnostica). Soluble thrombomodulin (STM) was measured by an ELISA (Diagnostica Stago, Asniéres, France). vWF antigen (vWF:Ag) was measured by latex immunoassay on a STAR coagulation instrument (Diagnostica Stago, Asnieres, France).

Thrombin-antithrombin complexes (TAT), markers of thrombin generation, were determined in citrated plasma using an ELISA (Siemens, Marburg, Germany). Two plasma platelet activation markers, i.e. soluble CD40 ligand (SCD4OL) and soluble P-selectin, were assessed by ELISA (R\&D Systems, Minneapolis, MN, USA).

Blood for clot lysis assay was collected into tubes containing $0.109 \mathrm{M}$ trisodium citrate and centrifuged within $20 \mathrm{~min}$ at $1500 \mathrm{~g}$. Plasma was frozen and stored at $-80^{\circ} \mathrm{C}$ until analysis. Technicians blinded to the origin of the samples performed all measurements. Intra-assay and inter-assay coefficients of variation were $<8 \%$.

\section{Clot lysis assay with and without TAFI inhibition}

Clot lysis time (CLT) was measured as described elsewhere in detail [12]. Briefly, to $75 \mathrm{ml}$ of citrated plasma we added TF (Innovin, dilution $10^{5}$ times), $\mathrm{CaCl}_{2}$ (final concentration, $17 \mathrm{mmol} / \mathrm{l}$ ), t-PA (final concentration, $30 \mathrm{U} / \mathrm{ml}$ ), and phospholipid vesicles (final concentration, $10 \mathrm{mmol} / \mathrm{l})$. HEPES buffer $(25 \mathrm{mmol} / \mathrm{l} \mathrm{HEPES}$, 
$137 \mathrm{mmol} / \mathrm{l} \mathrm{NaCl}, 3.5 \mathrm{mmol} / / \mathrm{KCl}, 3 \mathrm{mmol} / \mathrm{CaCl}_{2}, 0.1 \%$ bovine serum albumin, $\mathrm{pH}$ 7.4) was added to make a total volume of $150 \mathrm{ml}$. Phospholipid vesicles consisting of $40 \% \mathrm{~L}$-a-dioleoylphosphatidylcholine, $20 \%$ L-a-dioleoylphosphatidylserine, and 40\% L-a-dioleoylphosphatidylcholine (all from Sigma Chemical Co., St. Louis, MO) were prepared as described by Lisman et al. [14]. Total phospholipid content of the vesicles was determined by phosphate analysis according to Rouser et al. [15].

The solution was thoroughly mixed. Turbidity at $405 \mathrm{~nm}$ of $100 \mathrm{ml}$ of the mixture was measured in time at $37^{\circ} \mathrm{C}$ using a Spectramax 340 kinetic microplate reader (Molecular Devices Corp., Menlo Park, CA). The time from the midpoint of the clear to maximum turbid transition (clot formation) to the midpoint of the maximum turbid to clear transition (clot lysis) was defined as CLT. Contribution of TAFI to CLT was assessed in experiments by adding carboxypeptidase inhibitor from potato (CPI, Calbiochem, La Jolla, CA, final concentration, $25 \mathrm{mg} / \mathrm{ml}$ ), a specific inhibitor of activated TAFI [15], to the plasma.

\section{Statistical analysis}

Data are expressed as median (interquartile range), number (percentage), or mean (standard deviation) The Shapiro-Wilk test was used to determine normal data distribution. Student $t$ test was used to assess between-group differences for normally distributed continuous variables, and the Mann-Whitney $U$ test was used for variables of non-normal distribution. The Pearson correlation coefficient was used to evaluate simple linear relationships between variables. Clinical and laboratory variables that showed an association with CLT in the univariate model $(p<0.2)$ and did not show substantial correlations $(r>0.6)$ with another independent variable were included in the multiple regression analysis. The Hosmer-Lemenshow test was used to check the goodness of fit of the model. The results were presented after adjustment for clusters of confounding variables that are markers of different mechanisms: insulin resistance (BMI) and inflammation (fibrinogen and CRP). The significance level was set at $p<0.05$.

\section{Results}

\section{Patient characteristics}

There were no differences in age, sex, and cardiovascular risk factors between the patient and control groups [12]. The cancer group had higher CRP, fibrinogen, white blood cells (WBC) and platelets, higher INR and longer APTT, while albumin and protein were lower (Table I). Patients with lower grading (G1 + G2) compared with subjects with $\mathrm{G} 3$ tumours had higher BMI [12], albumin, and lower fibrinogen (Table I).

\section{Clot lysis}

In the DTC group CLT (by 22.3\%) and CLT CPI (by $27.4 \%$ ) were longer compared with control subjects (Table II), indicating that TAFI inhibition did not abolish the intergroup differences in lysis. Patients with higher grading $\mathrm{G} 3$ compared to those with grading $(\mathrm{G} 1+\mathrm{G} 2)$ had longer CLT and CLT CPI (Table II).

\section{Fibrinolysis parameters}

DTC patients had higher PAI-1 antigen, TAFI, both antigen and activity, compared with the control group (Table I). There was a non-significant trend toward lower tPA antigen in cancer patients. There were no differences between the groups in plasminogen and antiplasmin (Table I). Patients with G3 tumours, compared with those with lower grading $(G 1+G 2)$, had higher tPA antigen as well as TAFI activity and antigen (Table I).

\section{Other circulating markers}

The DTC group had higher SCD40L (by 438\%) and P-selectin (by 197\%) compared with controls (Table I), indicating increased platelet activation. Cancer patients had also higher thrombin generation in plasma as reflected by TAT, combined with enhanced endothelial injury as reflected by higher STM and VWF antigen compared to the control group. High-grade tumour patients had higher sTM and VWF compared with the lower-grade patient group (Table I).

\section{Associations}

The CLT correlated positively with tPA antigen, PAI-1, TAT, TAFI antigen and activity, and VWF (Table III) in cancer patients.

Multiple regression analysis showed that independent predictors of CLT in DTC patients after adjustment for potential confounders were TAT, TAFI activity, and VWF, while the only independent predictor of CLT CPI was plasma TAT concentration (Table IV).

There were no differences in demographics, clinical and laboratory variables, fibrinolysis parameters, platelet markers, clot lysis assay depending on the tumour type, and clinical stage of the disease (data not shown).

\section{Discussion}

The present study demonstrated a complex regulation of plasma clot lysis in patients with DTC. We found that reduced susceptibility to lysis can be observed both in the presence and absence of activated TAFI inhibitor (CPI), suggesting that TAFI is not the only major contributor to hypofibrinolysis in this disease. The CLT was reduced approximately by half by the addition of CPI, which confirms downregulation of fibrinolysis by TAFI in 
Table I. Laboratory investigations in digestive tract cancer (DTC) patients and control subjects - comparison of patients with G3 vs. G1 + G2 tumours of various cell differentiations

\begin{tabular}{|c|c|c|c|c|}
\hline Variable & Controls $(n=47)$ & DTC $(n=44)$ & $\mathrm{G} 1+\mathrm{G} 2(n=18)$ & $\mathrm{G} 3(n=26)$ \\
\hline \multicolumn{5}{|l|}{ Routine laboratory tests: } \\
\hline Albumin $[\mathrm{g} / \mathrm{l}]$ & $43.3 \pm 4.4$ & $39.5 \pm 7.7^{\circ}$ & $44(41-47)$ & $38(33-42)^{*}$ \\
\hline Protein [g/l] & $71.0 \pm 5.9$ & $67.7 \pm 8.8^{\circ}$ & $69.5(68-75)$ & $68(61-72)$ \\
\hline $\mathrm{CRP}[\mathrm{mg} / \mathrm{l}]$ & $1.8 \pm 1.0$ & $22.3 \pm 32.1^{\circ}$ & $5.5(3.7-12)$ & $16.5(1.9-36.4)$ \\
\hline INR & $0.99 \pm 0.1$ & $1.04 \pm 0.1^{0}$ & $1(1-1.1)$ & $1(1-1.1)$ \\
\hline APTT [s] & $27.7 \pm 2.6$ & $30.5 \pm 3.8^{\circ}$ & $28.35(27.7-29.5)$ & $31.4(28.7-32.9)^{\star}$ \\
\hline Fibrinogen $[\mathrm{g} / \mathrm{l}]$ & $3.3 \pm 1.0$ & $4.2 \pm 1.6^{0}$ & $3.51 \pm 0.9$ & $4.7 \pm 1.75^{\star}$ \\
\hline $\mathrm{WBC}\left[\times 10^{3} / \mu \mathrm{l}\right]$ & $6.7 \pm 1.3$ & $8.3 \pm 3.1^{\circ}$ & $7.6(5.7-8.7)$ & $8.3(6.4-11.1)$ \\
\hline $\mathrm{RBC}\left[\times 10^{6} / \mu \mathrm{l}\right]$ & $4.6 \pm 0.4$ & $5.4 \pm 0.64$ & $4.93(4.45-5.29)$ & $4.2(3.79-4.92)^{*}$ \\
\hline Haemoglobin [g/dl] & $13.8 \pm 1.2$ & $12.4 \pm 2.3^{\circ}$ & $12.9 \pm 2.6$ & $12.0 \pm 2.04$ \\
\hline Haematocrit (\%) & $41.4 \pm 3.2$ & $37.6 \pm 5.9^{\circ}$ & $39.3 \pm 6.1$ & $36.5 \pm 5.6$ \\
\hline Platelets $\left[\times 10^{3} / \mu \mathrm{l}\right]$ & $227.4 \pm 57.7$ & $302.3 \pm 127.5^{\circ}$ & $333(211-301)$ & $349.5(230-386)$ \\
\hline \multicolumn{5}{|l|}{ Fibrinolysis parameters: } \\
\hline Plasminogen (\%) & $102.51 \pm 10.6$ & $103.77 \pm 9.9$ & $101.39 \pm 9.95$ & $105.42 \pm 9.74$ \\
\hline$\alpha_{2} \mathrm{AP}(\%)$ & $101.47 \pm 11.36$ & $103.95 \pm 12.9$ & $100.5 \pm 12.5$ & $106.35 \pm 12.85$ \\
\hline $\mathrm{tPA}[\mathrm{ng} / \mathrm{ml}]$ & $10.03 \pm 2.18$ & $8.89 \pm 3.27$ & $6.98(5.47-9.9)$ & $9.15(6.8-12.5)^{*}$ \\
\hline PAl-1 [ng/ml] & $11.96 \pm 4.02$ & $14.14 \pm 5.91^{\circ}$ & $11.23(8.74-15.03)$ & $16.05(9.17-18.3)$ \\
\hline $\operatorname{TAFI}_{\mathrm{ACT}}[\mu \mathrm{g} / \mathrm{ml}]$ & $23.37 \pm 6.1$ & $27.42 \pm 5.98^{\circ}$ & $24.78 \pm 5.17$ & $29.24 \pm 5.9^{\star}$ \\
\hline TAFI $_{\text {ATG }}(\%)$ & $94.66 \pm 14.29$ & $105.27 \pm 13.44^{\circ}$ & $98.3 \pm 10.9$ & $110.08 \pm 13.1^{*}$ \\
\hline \multicolumn{5}{|l|}{ Platelet markers: } \\
\hline sCD40L [ng/ml] & $1.03(0.88-1.21)$ & $4.51(3.38-4.79)^{\circ}$ & $4.43 \pm 1.19$ & $4.25 \pm 0.92$ \\
\hline P-selectin [ng/ml] & 198 (178-214) & $390(298-467)^{\diamond}$ & $375.33 \pm 89.55$ & $387.58 \pm 132.34$ \\
\hline \multicolumn{5}{|l|}{ Other markers: } \\
\hline TAT [ $\mu \mathrm{g} / \mathrm{l}]$ & $4.9 \pm 1.52$ & $7.29 \pm 1.42^{\circ}$ & $6.45(6.2-7.3)$ & 7.35 (6.2-8.9) \\
\hline sTM [ng/ml] & $2.44 \pm 0.41$ & $2.86 \pm 1.24^{\circ}$ & $2.27 \pm 0.55$ & $3.19 \pm 1.46^{*}$ \\
\hline $\operatorname{VWF}[\mathrm{IU} / \mathrm{dl}]$ & $114.6 \pm 20.6$ & $184.5 \pm 38.4^{\circ}$ & $158.4 \pm 27.5$ & $202.6 \pm 34.5^{*}$ \\
\hline
\end{tabular}

Values are given as median (interquartile range), number (percentage), or mean \pm SD. CRP-C-reactive protein, INR - international normalized ratio, APTT activated partial thromboplastin time, WBC - white blood cells, RBC - red blood cells, tPA - tissue-type plasminogen activator, PAI-1 - plasminogen activator inhibitor-1, $\alpha$ AP - $\alpha_{2}$-antiplasmin, TAFI - thrombin activatable fibrinolysis inhibitor, SCD $40 L$ - soluble CD 40 ligand, TAT - thrombin-antithrombin complexes, STM - soluble thrombomodulin, vWF-von Willebrand factor antigen. ${ }^{\circ} p<0.05$ vs. controls. ${ }^{*} p<0.05$ vs. $(G 1+G 2)$.

Table II. Clot lysis time (CLT) and clot lysis time in the presence of carboxypeptidase potato inhibitor (CLT CPI) comparison of patients with digestive tract cancer (DTC) - G3 vs. G1 + G2 - and controls

\begin{tabular}{|c|c|c|c|c|}
\hline Variables & Controls $(n=47)$ & DTC $(n=44)$ & $\mathrm{G} 1+\mathrm{G} 2(n=18)$ & $\mathrm{G} 3(n=26)$ \\
\hline CLT [min] & $83.85 \pm 13.3$ & $102.6 \pm 20.1^{\circ}$ & $99.5 \pm 16.8$ & $109.62 \pm 19.4^{*}$ \\
\hline CLT CPI [min] & $42.14 \pm 6.4$ & $53.7 \pm 11.5^{\diamond}$ & $49.83 \pm 9.87$ & $56.38 \pm 12$ \\
\hline
\end{tabular}

Data are given as mean $\pm S D .{ }^{\circ} p<0.05$ vs. controls. ${ }^{*} p<0.05$ vs. $(G 1+G 2)$. 
Table III. Correlations of clot lysis time (CLT) and haemostatic parameters in cancer patients

\begin{tabular}{cccccccc} 
& tPA & PAI-1 & TAT & TAFI $_{\text {ACT }}$ & TAFI $_{\text {ATG }}$ & Antiplasmin & vWF \\
\hline CLT & $r=0.49$ & $r=0.49$ & $r=0.72$ & $r=0.82$ & $r=0.55$ & $r=0.53$ & $r=0.6$ \\
\cline { 2 - 7 } & $p=0.001$ & $p=0.001$ & $p<0.001$ & $p<0.001$ & $p<0.001$ & $p<0.001$ & $p<0.001$
\end{tabular}

Abbreviations see Table I.

Table IV. Independent predictors of clot lysis time (CLT) and clot lysis time in the presence of carboxypeptidase potato inhibitor (CLT CPI) in the cancer patients group - multiple stepwise regression analysis models after adjustment for potential confounders

\begin{tabular}{cccc} 
& & Independent predictors of CLT & TAT \\
\hline TAT & TAFl activity & vWF antigen & $R^{2}=0.58$ \\
\hline$R^{2}=0.84$ & & Slope estimate $=0.67$ \\
\hline Slope estimate $=0.28$ & Slope estimate $=0.49$ & Slope estimate $=0.25$ & $S E=0.88$ \\
\hline$S E=1.35$ & $S E=0.36$ & $S E=0.05$ & $p<0.001$
\end{tabular}

Abbreviations see Table I.

our patients. Elevated activity and antigen of TAFI were previously described in cancer patients [7-9], including DTC, which is consistent with this report. Elevated activated TAFI might be explained by higher thrombin formation, which increases TAFI activation occurring efficiently in complexes with TM, of which a soluble form was significantly increased in the DTC patients, suggesting that vascular injury/endothelial activation in cancer promote TM-mediated pro-and anticoagulant effects [16]. A novel finding is that TAFI activity is an independent predictor of CLT in the DTC patient group, which suggests a role of this inhibitor in the regulation of lysis in cancer. Moreover, our study provides evidence that patients with high-grade tumours (G3) have longer CLT compared with the lower grade cancer group (G1 + G2), which supports the concept that dysregulated haemostasis, including efficiency of fibrinolysis measurable in plasma of circulating blood, is more pronounced in more advanced malignant disease. In addition, elevated TAFI levels in the G3 group may lead to a hypothesis that in higher-grade tumours TAFI levels have a more potent role in fibrinolysis impairment compared to lower-grade cancer patients. This concept merits further in vitro investigation to elucidate the mechanisms of this observation.

Our current findings highlight the role of increased thrombin generation also in the degradation of plasma clots in cancer patients, probably in part by the formation of denser clots [17]. Previously we showed enhanced thrombin generation in DTC patients [12] Associations between clot lysis and thrombin generation have also been reported in patients with multiple myeloma [18] as well in those with other non-malignant prothrombotic disorders using different methodological approaches $[19,20]$. The current study unexpectedly demonstrated that thrombin generation, reflected by plasma TAT levels, can predict CLT following TAFI inhibition, suggesting a potent impact of thrombin on clot structure and/or efficiency of lytic mechanisms in this clinical setting. Since thrombin generation has been shown to predict thromboembolism in various malignant diseases by Ay et al. [21], our observations might support the concept indicating that this harmful effect of thrombin could be in part mediated by hypofibrinolysis. A larger patient group with long-term follow-up is needed to assess the links among thrombin, TAFI, fibrinolysis, and clinically overt thromboembolic manifestations in cancer patients.

Interestingly, we showed that the plasma vWF level correlates with CLT and is an independent predictor of CLT in DTC patients. This novel finding suggests that vWF is actively involved in clot fibrinolysis in cancer patients. Two major functions of vWF, the largest human plasma protein, are its involvement in platelet adhesion and carriership of factor VIII in circulation. Several studies have demonstrated increased plasma vWF antigen levels in malignancies, including CRC [22], which is in line with our findings. It is unclear whether there are associations between vWF level, fibrinolysis, and cancer growth. Damin et al. [23] reported associations between plasma vWF levels and tumour staging, invasion of adjacent organs by the tumour, and the presence of distant metastases in CRC. We found that patients with G3 tumours ( $n=26$ ), compared to those with lower grading 
$(n=18)$, had higher plasma vWF antigen levels. However, we failed to show such correlations, which might be because of the cancer type heterogeneity in the present DTC group. Interestingly, we found that CLT is positively associated with vWF antigen, which might suggest that this protein alters the efficiency of plasmin-mediated lysis, probably in part by affecting fibrin clot structure. This novel association and the potential role of VWF in modulation of thrombus properties merit further investigation given the multiple prothrombotic non-cancer disorders in which vWF levels rise substantially

We have also hypothesised that enhanced platelet activation in DTC patients might be involved in hypofibrinolysis. The rationale for this concept is derived from reports showing that platelet activation, which leads to the release of a number of substances, including proteins from $\alpha$ granules (e.g. platelet factor 4 (PF4), factor V, vWF, fibrinogen, angiogenin, vascular endothelial growth factor) and dense granules (predominantly various polyphosphate) unfavourably alters clot characteristics [24]. Elevated platelet counts in subjects with chronic inflammatory diseases have been reported to be associated with unfavourably altered clot properties, including a positive correlation with CLT [25]. We found that plasma platelet markers levels, P-selectin and SCD40L, are elevated in the cancer group, which is consistent with previous findings [26, 27]. The present study showed no association between CLT and these platelet markers, indicating a minor, if any, role of P-selectin and SCD40L in clot structure and function in cancer patients.

Our study has several limitations. There were a small number of patients enrolled in the study. However, our study was sufficiently powered to show intergroup differences, although the subgroup analysis, especially regarding subjects with various grades, should be interpreted with caution. Variables were determined at a single point in time. It would be of interest to assess post-treatment fibrin clot analysis and repeat the measurements after follow-up; however, treatment-associated alteration of CLT and the potential association with clinical outcome were beyond the scope of the current study. Fibrinolysis in cancer patients could also be modified by several environmental factors not specifically addressed by the present study, for instance active or passive tobacco smoking [28]. Finally, the associations reported in the study do not necessarily indicate cause-effect relationships.

\section{Conclusions}

We showed that TAFI, thrombin generation, and $\mathrm{VWF}$, commonly elevated in cancer patients, are involved in hypofibrinolysis assessed using a global plasma-based assay in the DTC group. Our findings, by showing novel potential determinants of slower fibrin degradation in cancer patients, provide new insights into the disturbed balance between blood coagulation and fibrinolysis in cancer patients, which is highlighted by more pronounced impairment of clot lysis in subjects with more advanced cancer. It remains to be established whether these alterations might represent an important thromboembolic risk factor in these types of malignancy.

\section{Acknowledgments}

This work was supported by the Jagiellonian University Medical College (K/ZDS/002936, to A.U.).

\section{Conflict of interest}

The authors declare no conflict of interest.

\section{References}

1. Gross CP, Galusha DH, Krumholz HM. The impact of venous thromboembolism on risk of death or hemorrhage in older cancer patients. J Gen Intern Med 2007; 22: 321-6.

2. Ay C, Dunkler D, Pirker R, et al. High D-dimer levels are associated with poor prognosis in cancer patients. Haematologica 2012; 97: 1158-64.

3. Raj SD, Zhou X, Bueso-Ramos CE, et al. Prognostic significance of elevated D-dimer for survival in patients with sarcoma. Am J Clin Oncol 2012; 35: 462-7.

4. Kockar C, Kockar O, Ozturk M, et al. Global fibrinolytic capacity increased exponentially in metastatic colorectal cancer. Clin Appl Thromb Hemost 2004; 11: 227-30.

5. Ferroni P, Roselli M, Portarena I, et al. Plasma plasminogen activator inhibitor-1 (PAI-1) levels in breast cancer - relationship with clinical outcome. Anticancer Res 2014; 34: 1153-61.

6. Koldas M, Gummus M, Seker M, et al. Thrombin-activatable fibrinolysis inhibitor levels in patients with non-small-cell lung cancer. Clin Lung Cancer 2008; 9: 112-5.

7. Hataji O, Taguchi O, Gabazza EC, Yuda H, et al. Increased circulating levels of thrombin-activatable fibrinolysis inhibitor in lung cancer patients. Am J Hematol 2004; 76: 214-9.

8. Fidan E, Kavgaci H, Orem A, et al. Thrombin activatable fibrinolysis inhibitor and thrombin-antithrombin-III-complex levels in patients with gastric cancer. Tumour Biol 2012; 33: 1519-25.

9. Cesarman-Maus G, Hajjar KA. Molecular mechanisms of fibrinolysis. Br J Haematol 2005; 129: 307-21.

10. Abe J, Urano T, Konno H, et al. Larger and more invasive colorectal carcinoma contains larger amounts of plasminogen activator inhibitor type 1 and its relative ratio over urokinase receptor correlates well with tumor size. Cancer 1999; 15: 2602-11.

11. Langenskiold M, Holmdahl L, Angenete E, et al. Differential prognostic impact of UPA and PAI-1 in colon and rectal cancer. Tumor Biology 2009; 30: 210-20.

12. Gronostaj K, Richter P, Nowak W, Undas A. Altered plasma fibrin clot properties in patients with digestive tract cancers: links with the increased thrombin generation. Thromb Res 2013; 131: 262-7. 
13. Edge SB, Byrd DR, Compton CC, et al. Purposes and principles of cancer staging. In: AJCC Cancer Staging Manual, 7th ed. Springer, New York, 2010; 3-14.

14. Lisman T, Leebeek FW, Mosnier LO, et al. Thrombin-activatable fibrinolysis inhibitor deficiency in cirrhosis is not associated with increased plasma fibrinolysis. Gastroenterology 2001; 121: 131-9.

15. Rouser G, Fkeischer S, Yamamoto A. Two dimensional thin layer chromatographic separation of polar lipids and determination of phospholipids by phosphorus analysis of spots. Lipids 1970; 5: 494-6.

16. Colucci M, Binetti BM, Tripodi A, et al. Hyperprothrombinemia associated with prothrombin G20210A mutation inhibits plasma fibrinolysis through a TAFI-mediated mechanism. Blood 2004; 103: 2157-161.

17. Undas A. Fibrin clot properties and their modulation in thrombotic disorders. Thromb Haemost 2014; 112: 32-42.

18. Undas A, Zubkiewicz-Usnarska L, Helbig G, et al. Altered plasma fibrin clot properties and fibrinolysis in patients with multiple myeloma. Eur J Clin Invest 2014; 44: 557-66.

19. Konieczynska M, Fil K, Bazanek M, Undas A. Prolonged duration of type 2 diabetes is associated with increased thrombin generation, prothrombotic fibrin clot phenotype and impaired fibrinolysis. Thromb Haemost 2014; 111: 685-93.

20. Gajos G, Zalewski J, Rostoff P, et al. Reduced thrombin formation and altered fibrin clot properties induced by polyunsaturated omega-3 fatty acids on top of dual antiplatelet therapy in patients undergoing percutaneous coronary intervention (OMEGA-PCI clot). Arterioscler Thromb Vasc Biol 2011; 31 1696-702.

21. Ay C, Dunkler D, Simanek R, et al. Prediction of venous thromboembolism in patients with cancer by measuring thrombin generation: results from the Vienna Cancer and Thrombosis Study. J Clin Oncol 2011; 29: 2099-103.

22. Schellierer VS, Mueller-Berg L, Merkel S, et al. The clinical value of von Willebrand factor in colorectal carcinomas. Am J Transl Res 2011; 3: 445-53.

23. Damin DC, Rosito MA, Gus P, et al. Von Willebrand factor in colorectal cancer. Int J Colorectal Dis 2002; 17: 42-5.

24. Kwasny-Krochin B, Gluszko P, Undas A. Unfavorably altered fibrin clot properties in patients with active rheumatoid arthritis. Thromb Res 2010; 126: 11-6.

25. Franchini M, Frattini F, Crestani S, et al. Von Willebrand factor and cancer: a renewed interest. Thromb Res 2013; 131: 290-2.

26. Ay C, Simanek R, Vormittag R, et al. High plasma levels of soluble P-selectin are predictive of venous thromboembolism in cancer patients: results from the Vienna Cancer and Thrombosis Study (CATS). Blood 2008; 112: 2703-8.

27. Hock BD, McKenzie JL, Patton NW, et al. Circulating levels and clinical significance of soluble CD40 in patients with hematologic malignancies. Cancer 2006; 106: 2148-57.

28. Szpak D, Grochowalski A, Chrząszcz R, et al. Tobacco smoke exposure and endothelial dysfunction in patients with advanced coronary artery disease. Pol Arch Med Wewn 2013; 123: 474-81.

Received: 8.01.2015

Accepted: 18.04.2015 